\title{
The effect of betel nut chewing on contour and object masking
}

\author{
Ming-Chou Ho • Chin-Kun Wang
}

Published online: 6 October 2011

(C) Psychonomic Society, Inc. 2011

\begin{abstract}
The betel nut is a common stimulant in many Asian countries. We employed the masking task developed by Enns and Di Lollo (Trends in Cognitive Sciences, 4, $345-352,1997)$ to investigate the effects of betel nuts on sensory and attentional processing. In the masking task, participants needed to identify a target that was masked by either a contour mask or an object mask. Sensory processing was assessed by examining target identification in the contour mask condition when the target was presented only centrally, whereas attentional processing was assessed by examining target identification in the object mask condition when the target was presented randomly in either a central or a parafoveal location. The results showed that chewing betel nut and chewing gum produced significant contour masking with a large effect size, similar to the pure control condition, in which participants chewed nothing, and the placebo control condition, in which what participants chewed was disguised. This suggests that neither betel nut nor gum affects sensory processing. Alternatively, betel nut chewing could produce a reduction in object masking for the habitual chewers and the nonchewers, suggesting an effect of betel nut on attentional processing. This concen-
\end{abstract}

M.-C. Ho $(\bowtie)$

Department of Psychology, Chung-Shan Medical University,

No. 110 , Sec. 1, Chien-Kuo N. Road,

Taichung 402, Taiwan

e-mail: mingchou@csmu.edu.tw

M.-C. Ho

Clinical Psychological Room,

Chung-Shan Medical University Hospital,

Taichung, Taiwan

C.-K. Wang

School of Nutrition, Chung-Shan Medical University,

Taichung, Taiwan trated attention was also observed in the placebo control condition; thus, it cannot be exclusively driven by the expectation effect. Also, chewing per se reduced the attentional distribution foveally.

Keywords Betel nut $\cdot$ Areca $\cdot$ Masking Contour $\cdot$ Object . Stimulant effects

Betel nut (also known as areca) is the fourth most commonly used drug worldwide, after tobacco, alcohol, and caffeine (Winstock, 2002; Winstock, Trivedy, Warnakulasuriya, \& Peters, 2000). A recent survey suggested that over 600 million people worldwide chew betel nuts (Gupta \& Warnakulasuriya, 2002). About 1.5 million Taiwanese are betel nut users, with about $30 \%$ of these users chewing betel nuts for refreshment purposes (Directorate-General of Budget, Accounting and Statistics, 1999). People place a whole betel nut into their mouths and macerate it by biting for approximately 2-3 $\mathrm{min}$; they then spit out the saliva, which has turned red from chewing the betel nut.

A betel nut usually consists of three major ingredients: a raw areca nut, slaked lime, and a piper betel flower. The primary chemical ingredients in betel nuts are alkaloids (i.e., arecoline, arecaidine, guvaeoline, guvacine, and acolidine), polyphenolic compounds, safrole, eugenol, and hydroxychavicol (Hwang, Wang, \& Kao, 1993). Arecoline, the primary alkaloid in the betel nut, acts as an agonist primarily at muscarinic acetylcholine receptors (Chu, 2001, 2002; Taylor, 1980; Von Euler \& Domeij, 1945; Winstock, 2002) and stimulates release of acetylcholine (Haubrich \& Watson, 1972; Molinengo, Fundaro, \& Cassone, 1988). Animal studies have shown that injection of arecoline evokes activations in many cortical (e.g., Haubrich \& Watson, 1972; Rinaldi \& Himwich, 1955) and subcortical 
areas (e.g., Molinengo et al., 1988) involved in attention (e.g., frontal cortex), sensory processing (e.g., visual cortex) and memory (e.g., hippocampus and limbic areas; for a review, see Freo, Pizzolato, Dam, Ori, \& Battistin, 2002). Human studies have shown that acetylcholine improves many cognitive functions (for reviews, see Clader \& Wang, 2005, and Freo et al., 2002). For example, in patients with Alzheimer's disease, arecoline has been shown to improve patients' attention, visuospatial performance, verbal ability, and memory (Asthana et al., 1995; Raffaele, Berardi, Haxby, Morris, Schapiro, \& Soncrant, 1996; Raffaele, Berardi, Morris, Asthana, Haxby, Schapiro, \& Soncrant, 1991). An electroencephalographic (EEG) study with betel nut chewers (Chu, 1994) suggested that betel nut chewing causes EEG changes associated with a state of arousal. Both an increase in beta and a decrease in theta waves indicated an increase in the state of arousal, whereas an increase in alpha waves indicated an increase in relaxation or calmness while chewing betel nuts.

In addition to the effects on the central nervous system, betel nut chewing also causes changes in the autonomic nervous system, mainly a sympathetic activation (for reviews, see Chu, 1995, 2001; Javed, Correa, Chotai, Tappuni, \& Almas, 2010). For example, the betel nut has been shown to increase the heart rate in naive, occasional, and habitual chewers (Chiou \& Kuo, 2008; Chu, 1993). Furthermore, percentage changes in heart rate following betel nut consumption in naive and occasional chewers are higher than changes in habitual chewers, suggesting that habitual chewers are tolerant of the cardiovascular response to betel nut chewing (Lin, Chang, Ryu, \& Chu, 2002).

Evidence that supports the effect of arecoline on physiology and cognition comes primarily from animal and Alzheimer studies in which participants are not required to consume the betel nut. Therefore, it remains unclear whether chewing betel nut affects healthy betel nut chewers. As far as we know, the present study is the first to address this question. This question is of practical importance, given that many people chew betel nut for refreshment purposes (Directorate-General of Budget, Accounting and Statistics, 1999). For example, a recent survey by Chen, Huang, Song, and Chang (2003) reported that $20.9 \%$ of bus drivers were betel nut users. If chewing betel nut can influence attention, it might have consequences for a variety of daily activities, including driving.

The animal studies showed that arecoline could affect many cortical and subcortical areas involved in sensory processing. It was therefore hypothesized that betel nut chewing might immediately increase the chewers' and nonchewers' sensitivity to sensory processing. Alternatively, because the studies supporting the effect of arecoline on sensory processing are primarily animalbased, it remains possible that betel nut chewing may not affect human chewers' sensory processing. For example, the amount of arecoline used in the animal studies (e.g., Freo et al., 2002) may be larger than that contained in one betel nut chewed by an adult chewer, so that it remains possible that betel nut chewing may not affect human chewers' sensory processing.

With regard to attentional processing, since previous animal and Alzheimer studies suggested that arecoline could facilitate an increase in focused attention, we hypothesized that chewing betel nuts could facilitate such an increase in the chewers' capacity to focus their attention. Moreover, the studies of the autonomic nervous system mentioned above led us to hypothesize that betel nut chewing might have different effects on attention-focusing capacities for habitual chewers and nonchewers. Cardiac acceleration is associated with enhanced attentional selectivity, thus concentrating attention on relevant stimuli and rejecting irrelevant stimuli that distract performance (for reviews, see Critchley, 2005; Eysenck, 1982; Hugdahl, 1996; Laumann, Gärling, \& Stormark, 2003). Since the percentage increase in heart rate after betel nut consumption has been reported to be larger in nonchewers than in habitual chewers (Lin et al., 2002), we also hypothesized that chewing betel nuts could result in varied degrees of a heightened focus of attention in nonchewers and in habitual chewers. More specifically, chewing betel nut might focus nonchewers' attention strongly and habitual chewers' attention moderately.

One of the tasks used to distinguish between sensory and attentional processing is visual masking (e.g., Atchley, Grobe, \& Fields, 2002; Breitmeyer \& Öğmen, 2006; Enns, 2004; Enns \& Di Lollo, 1997). Visual masking refers to "the reduction of the visibility of one stimulus, called the target, by a spatiotemporally overlapping or contiguous second stimulus, called the mask" (Breitmeyer \& Öğmen, 2006, p.2). In this study, we primarily employed two types of masks, contour and object masks, to investigate sensory and attentional processing, respectively.

A contour mask refers to a mask that appears temporally before or after the target stimulus and that forms a contour around the stimulus, although it does not occupy the same spatial positions. Contour masking can be influenced by a variety of physical attributes, such as proximity (Enns \& Di Lollo, 1997), contrast (Macknik \& Livingstone, 1998), and background luminance (Stewart \& Purcell, 1974). In addition to the physical attributes, contour masking can be affected by attentional distribution (Enns, 2004; Ramachandran \& Cobb, 1995). For example, contour masking is more effective when attention is distributed over multiple target locations rather than over only one target location (Enns, 2004, Exp.1). When the target and mask are in close temporal proximity and when attention is directed to a single target, contour masking is insensitive to attention. In this case, contour 
masking is influenced primarily by temporal integration and local contour interactions. Therefore, the present study investigated sensory processing primarily through the observation of contour masking at a 50-ms target-to-mask stimulus onset asynchrony (SOA), which was little affected by attention.

The object mask is even simpler than the contour mask. For example, four dots surrounding the target stimulus usually serve as an object mask. The object mask could act as a powerful mask under conditions of spatial uncertainty (Enns \& Di Lollo, 1997). In such conditions, the target appears in one of multiple possible locations; thus, attention needs to be distributed over all of the possible locations in expectation of the target. Object masking could also be influenced by low-level sensory processing. Enns and Di Lollo (1997) suggested that an object mask may act as a camouflage mask at the zero-SOA point, indicating degradation of the target representation at this point through the addition of noise interference from the mask. At positive target-to-mask SOAs (particularly at a 50-ms SOA), object masking is sensitive to the attentional distribution over the display (Atchley et al., 2002; Enns, 2004; Enns \& Di Lollo, 1997). Therefore, the present study investigated attentional processing primarily through the observation of object masking at 50-ms target-to-mask SOAs.

The classic study by Enns and Di Lollo (1997, Exp. 1) compared contour and object masks and found several important differences. They presented either a contour or an object mask randomly at various values of SOA, ranging from $-300 \mathrm{~ms}$ (mask preceded target) to $+300 \mathrm{~ms}$ (target preceded mask). The target was a diamond with a missing corner on either the left or the right side. Participants were instructed that their responses should correspond to the missing corner. In one condition, called the one-location condition, the mask and target were always at the center of the display; in the other condition, called the three-location condition, the mask and target could each randomly appear centrally or parafoveally (to either the left or right of the center). The latter condition created spatial uncertainty.

Enns and Di Lollo (1997) found that when a contour mask trailed by $0-100 \mathrm{~ms}$, the target visibility decreased, although target visibility increased once again when the target-to-mask SOA was lengthened. On the other hand, the object mask did not reduce target visibility in the onelocation condition. In the three-location condition (spatial uncertainty), the contour mask reduced target visibility when the target was presented centrally (at the central fixation) and parafoveally (near the central fixation). However, the object mask impaired target visibility only when the target was parafoveal. The degraded attentional distribution at the parafoveal locations might have made target recognition less efficient; the masking thus occurred when the four dots substituted themselves for the decaying target representation before target recognition had been completed.

We applied the masking paradigm used in Enns and Di Lollo (1997, Exp.1) to examine how betel nut chewing affects sensory and attentional processing. If betel nut chewing immediately increases the sensitivity of sensory processing in the one-location condition, one would expect to see an increase in contour masking after betel nut chewing. If betel nut chewing can immediately facilitate attention, one would predict a decrease in object masking in the three-location condition after betel nut chewing. Moreover, a placebo control condition was added to examine the possible expectation effect. Finally, a pure control was added to examine whether chewing per se had any effect on sensory and attentional processing.

\section{Method}

The present study had three experimental conditions: the treatment condition, the placebo control condition, and the pure control condition. In the treatment condition, participants (habitual chewers and nonchewers) were asked to chew betel nuts or gum before proceeding to the masking task. In the placebo control condition, since the habitual chewers were most likely to expect to experience effects when they chewed betel nuts (e.g., increased stamina), only the habitual chewers participated in this condition. Participants in this condition were asked to chew gum that was injected with a solution of sodium citrate with betel nut extract (hereafter, gum with extract) or with a solution of sodium citrate without betel nut extract (hereafter, gum without extract). Finally, only the nonchewers participated in the pure control condition, in which they chewed nothing.

\section{Participants}

In the treatment condition, 20 habitual betel nut chewers (all male; mean age $=35$ years, $S D=9.6$, range $=25-$ 58 years) and 18 nonchewers ( 5 females, 13 males; mean age $=33$ years, $S D=8.6$, range $=25-58$ years) participated. For the chewers, the average months of chewing betel nut were $103(S D=87$, range $=3-276)$, and the average number of days per week on which chewing occurred was $4(S D=2$, range $=1-7)$. The average number of betel nuts chewed per day was $14(S D=$ 12 , range $=1-50)$. In the placebo control condition, 12 habitual betel nut chewers ( 1 female; mean age $=34$ years, $S D=10.6$, range $=20-58$ years) participated. The average number of months of chewing betel nuts was $84(S D=91$, range $=3-360)$, and the average number of days per week 
on which chewing occurred was $5(S D=2$, range $=2-7)$. The average number of betel nuts chewed per day was 16 $(S D=14$, range $=2-50)$. In the pure control condition, 15 nonchewers (3 female; mean age $=34$ years, $S D=8.5$, range $=24-52$ years) participated.

There was no age difference between these four groups of participants $[F(3,66)=0.233, p>.8]$. All participants had normal or corrected-to-normal vision.

\section{Apparatus}

We used an IBM-compatible PC with a 17-in. CRT desktop monitor (refresh rate $=85 \mathrm{~Hz}$ ).

General design in three conditions

The stimuli were derived from those used by Enns and Di Lollo (1997). All of the stimuli were black on a white background (Fig. 1). The target was a diamond $\left(0.62^{\circ}\right.$ in vertical axis) with a missing corner $\left(0.17^{\circ}\right)$ on either the right or left side. The contour mask was a frame $\left(0.20^{\circ}\right.$ in width $)$ surrounding the target ( 1 pixel from the target). The object mask consisted of four squares $\left(0.20^{\circ}\right)$ placed on a notional square $\left(1.0^{\circ}\right.$ on each side). The minimum separation between neighboring contours in the target and mask was $0.35^{\circ}$. Two short vertical lines $\left(2.0^{\circ}\right.$ above and below the location of the central stimulus) served as the fixation point.

Each participant underwent three tasks in sequence. The tasks were administered in a dimly lit room where each participant leaned his or her chin on a chinrest with a fixed viewing distance of $50 \mathrm{~cm}$ from the monitor. In the first task (target identification, hereafter), the single target was presented centrally on the screen to familiarize the participants with the identification task. There were 36 trials in the first task. In the second task (one-location condition, hereafter), the target and mask were presented centrally. The contour and object masks appeared equally often and were randomly assigned across trials. There were 160 trials in the second task (10 per SOA and mask type). In the third task (three-location condition, hereafter), the target and mask were each assigned equally often and randomly to three horizontally arrayed locations, one central and two parafoveal $\left(3.0^{\circ}\right.$ to the left and right of center). The target and the mask appeared in the same location on one third of the trials and in different locations on two thirds of the trials. There were 288 trials for the third task (in which both the target and the mask were colocated; 2 and 4 trials per SOA and mask type for the central and parafoveal target conditions, respectively). The duration of both the target and mask was $32 \mathrm{~ms}$ in all tasks. The mask was presented at one of eight SOAs $(-150,-100$, $-50,0,50,100,150$, or $300 \mathrm{~ms}$ relative to the target). Before each task, there were 20 practice trials.

In the treatment condition, each participant underwent two conditions (gum and betel nut) that were counterbalanced across participants, so that half of the participants took part in the gum condition first, and the remaining half took part in the betel nut condition first. The gum condition was adopted in order to control for the effect of mere chewing. The chewing gum we used in the present study had a different taste, texture, and color from the betel nuts. The chewing gum we used was a small white cube of a size similar to that of a betel nut. The chewing gum tasted sweet and was not as tough as the betel nut. The two conditions
Fig. 1 Sample trial sequences in the present study. In the onelocation and three-location conditions, the target could precede the mask (as shown) or follow the mask. The mask shown in the one-location condition is a contour mask, and that shown in the three-location condition is an object mask
Target identification

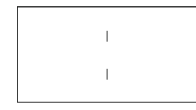

Fixation

(1000ms)

One-location condition

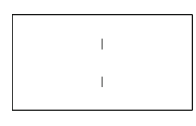

Fixation

(1000ms)

Three-location condition

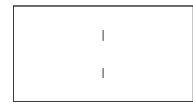

Fixation

(1000ms)

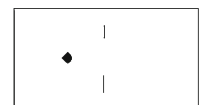

Target

(32 ms)

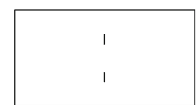

Various time intervals intervals

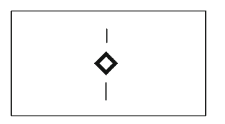

Mask (32 ms)

Until response

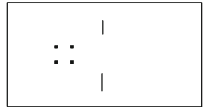

Mask (32 ms)
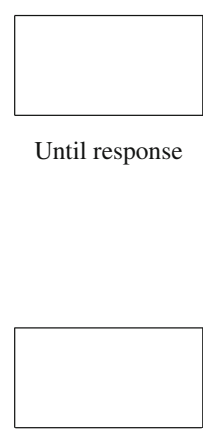

Until response 
were separated by no more than 1 week (habitual chewers: mean $=4$ days, $S D=3$, range $=1-7$ days; nonchewers: mean $=3$ days, $S D=2$, range $=1-7$ days). The laboratory prepared the betel nuts and chewing gum so that all of the participants chewed the same type of betel nuts and chewing gum.

In the placebo control condition, the design was similar to the treatment condition, except for the chewing substance. Since it is extremely difficult to find a substance that matches the betel nut in appearance, smell, and taste without having the same psychopharmacological ingredients, we used the chewing gum and injected two different fillers to mask the betel nut. One of the fillers was a solution of $0.5 \%$ citric acid with betel nut extract (primarily arecoline) dissolved in it (i.e., gum with extract). The solution of $0.5 \%$ citric acid was edible and could mask the taste and smell of betel nut extract. The arecoline was extracted from 60 betel nuts ${ }^{1}$ and weighed $80.2 \mathrm{mg}$. The other filler was a solution of $0.5 \%$ sodium citrate without betel nut extract (i.e., gum without extract). Each filler was $2 \mathrm{ml}$ in volume, and each gum was injected with about $0.1 \mathrm{ml}$ of fillers ( $2 \mathrm{ml}$ divided by the expected sample size of 20). In the placebo condition, the betel nut was replaced with the gum with extract, and the chewing gum was replaced with the gum without extract. These two conditions (gum with and without extract) were separated by no more than 1 week (mean $=4$ days, $S D=2$, range $=1-7$ days).

In the pure control condition, because participants chewed nothing, they participated in the three masking tasks only once.

\section{Procedure}

In the three masking tasks, participants were instructed to press the mouse button corresponding to the missing corner of the target (left or right) that flashed briefly. They were also instructed to respond as accurately as possible, without worrying about the response speed. They were allowed to make their best guess if they were not sure of the correct answer. Participants were instructed to fixate on the central location between the vertical lines at the beginning of each trial. In addition to the general instructions, task-specific instructions were provided. In the second task, participants were informed that in addition to the target, one other figure would appear briefly. In the third task, participants were

\footnotetext{
${ }^{1}$ The number of betel nuts for extraction was equal to the expected number of participants (20) times the number (3) of betel nuts for each participant. Since some injected gums were consumed for a pilot test, some extracts were lost on the pipe wall and pipette during the injection procedure, and 5 participants failed the task, the actual sample size became 12 .
}

informed that the target and one other figure would each appear randomly at three locations. In each task, after practice and before the formal trials, participants were required to chew the substance (either gum or betel nut in the treatment condition, or gum with or without extract in the placebo control condition) for 3 min. Immediately after they spit out the substance, the formal trials began.

\section{Results and discussion}

General analytic procedure

The first component of the analysis concerned the establishment of a baseline in the one- and three-location conditions, as guided by Atchley and Hoffman (2004). Because neither the contour mask nor the object mask produced a forward masking effect at the two longest target-to-mask SOAs (-150 and $-100 \mathrm{~ms})$, performance in these two SOA conditions was averaged to serve as a baseline. This baseline was used to compare with the masking performance at the critical SOAs. The baseline in the one-location condition was computed for each group (habitual chewers vs. nonchewers) $\times$ mask type (contour vs. object) $\times$ treatment (betel nut vs. gum) condition, and the baseline in the three-location condition was computed for each group $\times$ mask type $\times$ treatment $\times$ target location (foveal vs. parafoveal) condition. In the three-location condition, the baseline was computed based on the trials on which the target and the mask were at the same location. The trials on which the target and the mask appeared at different locations could also serve as a baseline (see, e.g., Atchley et al., 2002). However, when the mask appeared at a location different from that of the target, attention might shift to the mask location rather than to the target location. Therefore, it was more advantageous and ideal to assess the masking effect when attention was at the target location and not moved to another location.

The second component of the analysis concerned the critical SOAs to be analyzed. Previous studies (e.g., Atchley et al., 2002; Atchley \& Hoffman, 2004; Enns, 2004; Enns \& Di Lollo, 1997, 2000) have shown maximum masking effects (contour and object masks) at a target-tomask SOA of $50 \mathrm{~ms}$. Therefore, planned comparisons were conducted to compare performance at the 50-ms SOA with the baseline in all conditions. Since the masking effects could also take place at other SOAs (e.g., Enns \& Di Lollo, 1997), post hoc comparisons of other SOAs were conducted where appropriate.

The final component of the analysis included the computation of effect size, Cohen's $d$ (Cohen, 1988), to estimate the masking effects. Cohen's $d$ was calculated as 
specified by Rosnow and Rosenthal (1996): $d=$ $\left(X_{1}-X_{2}\right) / \sqrt{\left(S_{1}^{2}+S_{2}^{2}\right) / 2}$ where $X_{1}$ is the mean of Group 1, $X_{2}$ is the mean of Group 2, $S_{1}$ is the standard deviation of Group 1, and $S_{2}$ is the standard deviation of Group 2. In general, an effect size of about 0.3 is regarded as small; 0.5 , moderate; and 0.8 , large. In the present study, Group 1 is the baseline condition, and Group 2 is the masking condition. Group 2 (masking condition) in the one-location condition represented the accuracy rates at all SOAs, and in the three-location condition, Group 2 represented the accuracy rates at all SOAs when the target and the mask were at the same locations.

\section{Target identification}

In the treatment condition, the accuracy rates for the nonchewers in the betel nut and gum conditions were both $.96[t(17)=0.4, p=.686]$; for the habitual chewers, accuracy in the betel nut condition was .96 , and accuracy in the gum condition was .94 [t(19) $=1.2, p=.237]$. In the placebo control condition, the accuracy rate for the gum with extract was .98 , and for the gum without extract was $.97[t(11)=0.3, p=.791]$. In the pure control condition, the accuracy rate was .99 . Since there was no accuracy difference between the betel nut and gum conditions, the performance in these two conditions was combined. There was no accuracy difference between these four groups of participants $[F(3,61)=1.708, p=.175]$, showing equivalent performance in target identification.

\section{Examining sensory processing in the one-location condition}

Contour masking Accuracy rates in the treatment, placebo control, and pure control conditions are shown in Fig. 2. Contour masking at the 50-ms SOA was analyzed primarily for investigating sensory processing. The analysis revealed a significant reduction in accuracy by contour masks in the treatment, pure control, and placebo control conditions. In the treatment conditions, the contour mask caused a significant accuracy reduction $(31 \%-35 \%$; see Fig. 2$)$ in target identification for the nonchewers and the habitual chewers after they chewed either the betel nut or gum [for the nonchewers: with gum, $t(17)=12.5, p<.0001, d=$ 2.95; with betel nut, $t(17)=6.2, p<.0001, d=2.13$; for the habitual chewers: with gum, $t(19)=8.6, p<.0001, d=$ 2.76; with betel nut, $t(19)=6.7, p<.0001, d=2.13$ ]. Moreover, because a significant accuracy reduction with a contour mask $(41 \%)$ was also obtained in the pure control condition, in which participants chewed nothing $[t(14)=11.0$, $p<.0001, d=3.98]$, it appeared that neither betel nut nor gum influenced sensory processing. After controlling for the expectation effect, similar result patterns were obtained. That
Contour mask:

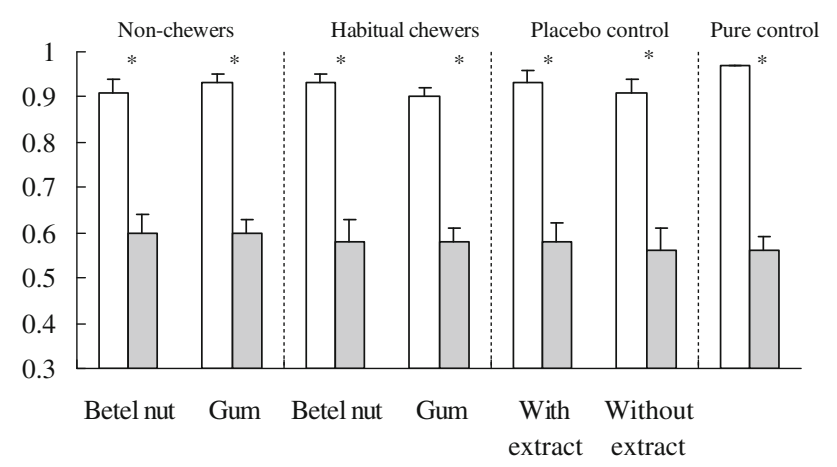

Object mask:

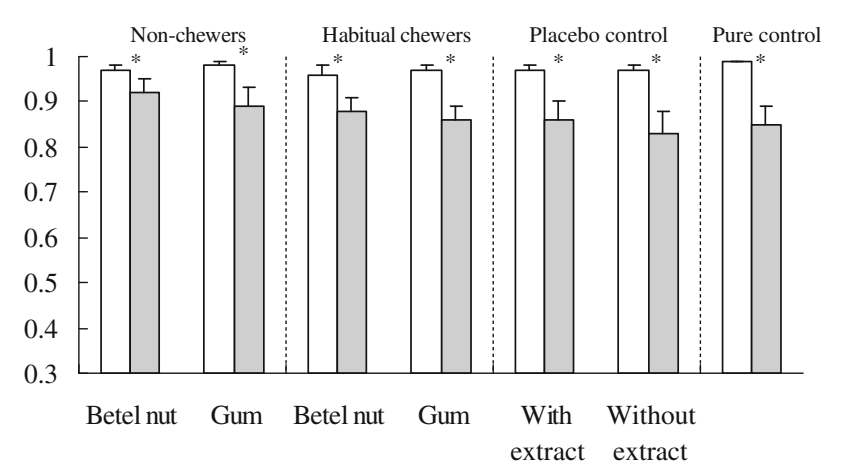

$\square$ Baseline $\square 50$-ms SOA

Fig. 2 Accuracy rates in the one-location condition for all groups of participants. Error bars indicate the standard errors of the means. Significant differences between the baseline and 50-ms-SOA conditions are denoted by asterisks

is, in the placebo control condition, a contour mask also caused an accuracy reduction of $35 \%$ after participants chewed either gum with extract $[t(11)=8.0, p<.0001$, $d=2.75]$ or gum without extract $[t(11)=6.5, p<.0001$, $d=2.62]$.

Object masking Finally, object masking at the same SOA was also found in the treatment, pure control, and placebo control conditions. The effect size of object masking was large across all conditions, but medium in the condition in which the nonchewers chewed betel nuts.

Examining attentional processing in the three-location condition

Accuracy rates in the treatment, placebo control, and pure control conditions are shown in Figs. 3 and 4. Object masking at the 50-ms SOA was analyzed primarily for investigating attentional processing. The analysis revealed the following primary findings. 
Contour mask, parafoveal target:

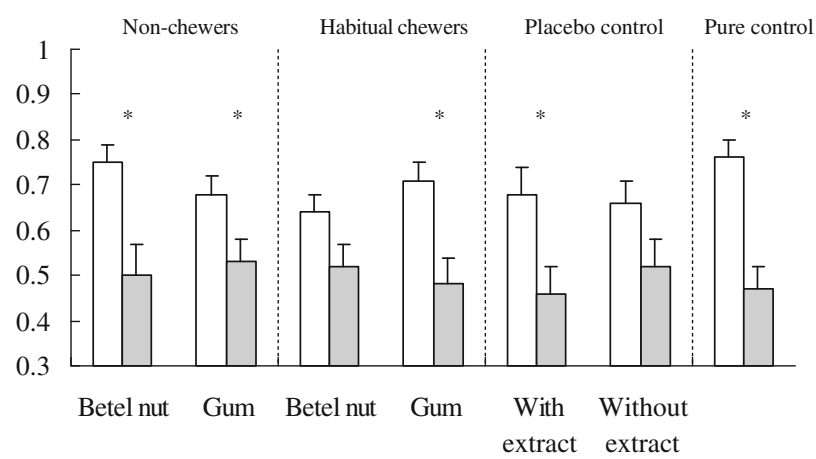

Contour mask, foveal target:

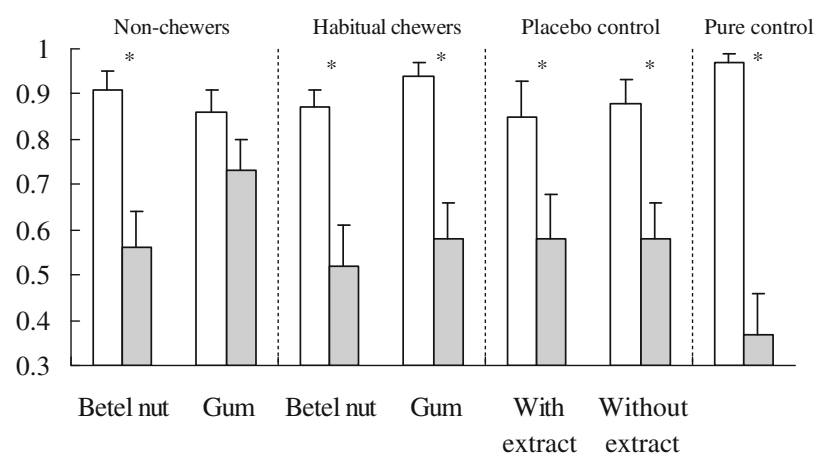

$\square$ Baseline $\square 50$-ms SOA

Fig. 3 Accuracy rates in the three-location condition for contour masks for all groups of participants. Error bars indicate the standard errors of the means. Significant differences between the baseline and 50-ms-SOA conditions are denoted by asterisks

First, betel nut chewing concentrated the nonchewers' attention foveally, thereby forming tunnel vision. For the nonchewers, an object mask caused a significant reduction in accuracy in the gum condition for the foveal target [14\% reduction; $t(17)=2.8, p<.05, d=0.72]$ and the parafoveal target [23\% reduction; $t(17)=3.5, p<.005, d=1.25]$. After the nonchewers had chewed betel nuts, the objectmasking effect disappeared for the foveal target $[t(17)=$ $0.9, p=.39, d=0.27]$ and remained significant for the parafoveal target $[27 \%$ reduction; $t(17)=4.2, p<.001, d=$ 1.38]. After the betel nut was consumed, the nonchewers concentrated their attention foveally, causing little, if any, masking effect foveally, and a large masking effect parafoveally. This indicates that betel nut chewing narrows nonchewers' attention immediately and results in the formation of tunnel vision.

Second, betel nut chewing could also concentrate the habitual chewers' attention foveally, but to a lesser degree. For the habitual chewers in the gum condition, there was a significant reduction in accuracy caused by an object mask for the foveal target [19\% reduction; $t(19)=2.9, p<.01, d=0.87]$ and for the parafoveal target $[24 \%$ reduction; $t(19)=4.6, p<$ $.0001, d=1.39$ ]. After the habitual chewers had chewed betel
Object mask, parafoveal target:

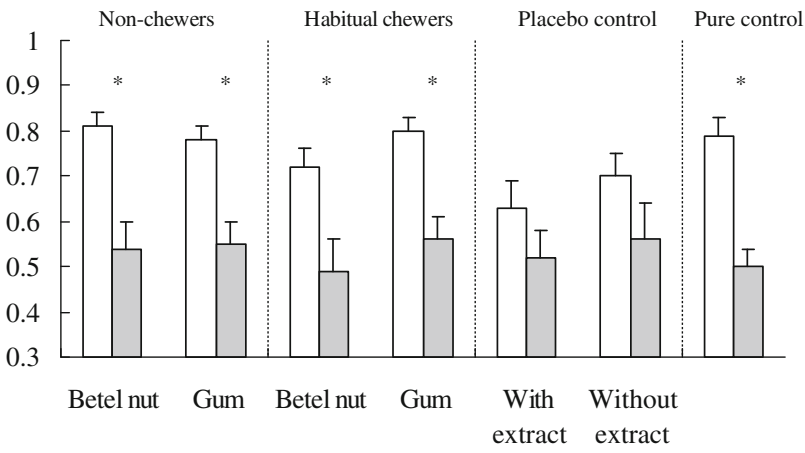

Object mask, foveal target:

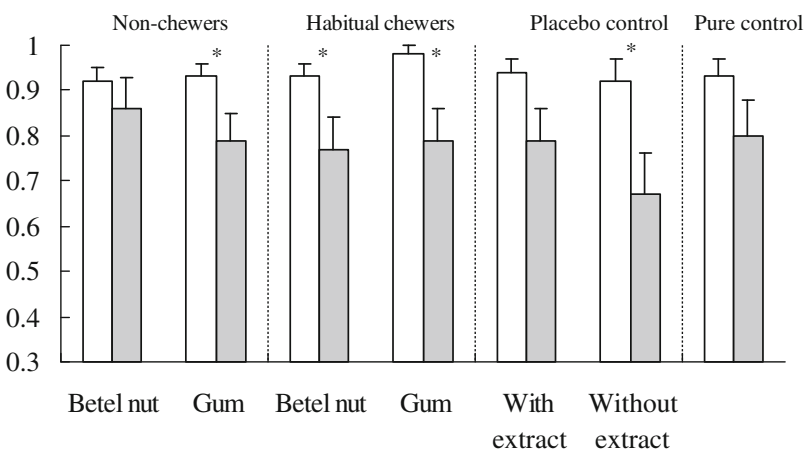

\section{$\square$ Baseline $\square 50-\mathrm{ms} \mathrm{SOA}$}

Fig. 4 Accuracy rates in the three-location condition for object masks for all groups of participants. Error bars indicate the standard errors of the means. Significant differences between the baseline and 50-msSOA conditions are denoted by asterisks

nuts, there was a significant reduction in accuracy for the foveal target [16\% reduction; $t(19)=2.5, p<.05, d=0.63$ ] and for the parafoveal target [23\% reduction; $t(19)=2.9, p<$ $.01, d=0.89]$. The moderate effect of object masking after the betel nut was consumed indicated facilitated attention in the foveal location. However, unlike the nonchewers, since this object-masking effect was statistically significant, the habitual chewers were less likely to experience tunnel vision.

Third, after controlling for the expectation effect, the overall effect on the size of object masking was reduced, but the facilitation effect for the habitual chewers described above still held. In the gumwithout-extract condition, there was a significant $25 \%$ reduction in accuracy for the foveal target $[t(11)=2.45$, $p<.05, d=0.96]$. In the gum-with-extract condition, this masking effect became nonsignificant for the foveal target $[t(11)=1.5, p=.15, d=0.75]$. Object masking after chewing the gum without extract became smaller after chewing the gum with extract. This indicates that when the habitual chewers were blind to what they chewed, their attention at the foveal location was facilitated after betel nut extract was consumed. 
In the placebo control, the object-masking effects at the parafoveal location were not significant [gum without extract, $t(11)=1.35, p=.20, d=0.61$; gum with extract, $t(11)=0.11, p=.21, d=0.49]$. This might have primarily been caused by the lower baseline means ( $63 \%$ in the gum with extract, and $70 \%$ in the gum without extract), given that the baseline means in the treatment condition and the pure control conditions were about $70 \%$ to $80 \%$.

Finally, chewing per se could reduce the attentional distribution at the foveal location. In the pure control condition, in which the nonchewers chewed nothing, an object mask caused a significant $29 \%$ accuracy reduction for the parafoveal target $[t(14)=5.8, p<.0001, d=1.93]$, but not for the foveal target $[t(14)=2.1, p=.056, d=0.52]$, showing that attention was allocated foveally. On the other hand, when the participants chewed gum in the treatment condition, or gum without extract in the placebo condition, an object mask caused a significant impairment in identifying a foveal target (see Figs. 3 and 4), showing less attention distributed foveally. Thus, it appears that chewing per se reduces attentional allocation foveally.

Contour masking at the 50-ms SOA was also analyzed. In the treatment condition, after consuming gum, the nonchewers showed a significant masking effect for the parafoveal target [15\% reduction; $t(17)=2.7, p<.05, d=$ $0.85]$, but this effect was not significant for the foveal target $[t(17)=1.6, p=.13, d=0.5]$. After the nonchewers had chewed betel nuts, contour masking was significant for the parafoveal target [25\% reduction; $t(17)=2.7, p<.05, d=$ $0.92]$ and for the foveal target [ $35 \%$ reduction; $t(17)=3.7$, $p<.005, d=1.29]$. Interestingly, for the nonchewers, contour masking was absent foveally in the gum condition, but present in the betel nut condition. It should be recalled that nonchewers narrowed down their attention to the foveal location after betel nut chewing. Possibly, contour masking in the three-location condition was sensitive to attentional distribution to some degree. Therefore, after betel nut chewing, the resulting increased attention at the foveal location could facilitate processing of physical attributes (e.g., edges and proximity), thus increasing contour masking foveally.

Notably, the attentional distribution in the three-location condition was unable to exclusively account for the contour masking effect (Enns, 2004; Enns \& Di Lollo, 1997); otherwise, contour masking should simply operate the same as object masking. Contour masking under conditions of spatial uncertainty (e.g., the three-location condition in the present study and the multiple-target conditions in Enns, 2004) is influenced by both sensory and attentional processing.

For the habitual chewers, a contour mask caused a significant accuracy reduction in target identification in the gum condition for the foveal target [36\% reduction; $t(19)=$
4.5, $p<.0001, d=1.4]$ and the parafoveal target $[23 \%$ reduction; $t(19)=3.1, p<.01, d=1.04]$. After the habitual chewers had chewed betel nut, a contour mask led to a significant reduction in accuracy for the foveal target $[35 \%$ reduction; $t(19)=3.7, p<.005, d=1.13$ ], but not for the parafoveal target $[t(19)=1.8, p=.096, d=0.6]$. After controlling for expectation in the placebo control condition, the result pattern was similar. When habitual chewers chewed the gum without extract, there was a significant reduction in accuracy by a contour mask for the foveal target [ $30 \%$ reduction; $t(11)=3.02, p<.05, d=1.26$ ], but not for the parafoveal target $[t(11)=1.47, p=.2, d=0.72]$. After chewing gum with extract, the habitual chewers showed a significant contour-masking effect for the foveal target [27\% reduction; $t(11)=2.24, p<.05, d=0.85]$ and for the parafoveal target [22\% reduction; $t(11)=2.67, p<$ $.05, d=1.05]$. In the pure control condition, in which the nonchewers chewed nothing, the contour-masking effects were significant for both foveal $[60 \%$ reduction; $t(14)=6.6$, $p<.0001, d=2.35]$ and parafoveal $[29 \%$ reduction; $t(14)=$ $5.9, p<.0001, d=1.62]$ targets.

\section{Discussion}

In the present study, sensory processing was assessed primarily by examining target identification in the contour mask condition at the 50-ms SOA when the target was presented only centrally (one-location condition). On the other hand, attentional processing was assessed primarily by examining target identification in the object mask condition when the target was presented randomly at a 50-ms SOA in foveal and parafoveal locations (three-location condition).

Two primary conclusions were drawn in the present study. First, significant contour masking with a large effect size was observed in the treatment, pure control, and placebo control conditions. We suggested that for both the habitual chewers and the nonchewers, neither the betel nut nor gum influenced sensory processing. One possibility to account for this null result may be that the small amount of arecoline in one betel nut may be insufficient to affect sensory processing. In the present study, since extraction of 60 betel nuts produced only $80.2 \mathrm{mg}$ of arecoline, speculatively one betel nut would contain only about $1.3 \mathrm{mg}$ arecoline. This amount of arecoline may be too little for an adult chewer, given that some animal studies (e.g., Freo et al., 2002) required $15 \mathrm{mg} / \mathrm{kg}$ to observe a widespread effect of arecoline on many brain areas involving sensory processing.

Second, betel nut chewing has an immediate effect on attentional processing for both habitual chewers and nonchewers. After chewing betel nuts, object masking was not significant for the nonchewers, whereas it was significant with moderate effect for the habitual chewers. This 
indicates that chewing betel nuts can focus the nonchewers' and the habitual chewers' attention foveally to different extents. That is, betel nut chewing focuses the nonchewers' attention strongly, possibly forming tunnel vision. Conversely, betel nut chewing focuses habitual chewers' attention only moderately. The facilitation effect caused by betel nut chewing could not be accounted for exclusively by expectation. In the placebo control condition, in which expectation was controlled, chewing a gum injected with betel nut extract reduced the degree of the effect of object masking at the foveal location, again showing that attention was facilitated.

In the present placebo control condition, because it was extremely difficult to find a substance similar in many respects to betel nut (e.g., taste, texture, and color), we used gum injected with different fillers. Although the present design was different from the typical placebo control design, it was appropriate for examining the expectation effect. If facilitated attention after betel nut chewing in the treatment condition were completely driven by the habitual chewer's expectation, one would predict similar objectmasking patterns between the gum-with-extract and gumwithout-extract conditions. Alternatively, if the facilitated attention after chewing betel nut derived from the joint effect of expectation and physiology, one would expect different object-masking patterns to arise from the gumwith-extract and gum-without-extract conditions. The latter (different object-masking patterns) was exactly what we found, suggesting that the primary ingredient (i.e., arecoline) of the betel nut can facilitate the focusing of attention.

The present study, using a behavioral approach, does not directly answer which neural mechanism of attentional control is affected by betel nut chewing for the chewers. As far as we know, the existing literature reports that arecoline has widespread effects on the central and autonomic nervous systems (e.g., Chu, 2001; Freo et al., 2002); therefore, the neural mechanism of attentional control affected by the betel nut should be complex, and would require more studies aimed directly at the neural mechanisms. Moreover, most of such physiology-focused studies have come from the animalrelated literature. Thus, future studies could use brainimaging techniques (e.g., functional magnetic resonance imaging) to investigate which brain areas are related to betel nut chewing for healthy adult chewers.

The present finding of facilitated attention due to the betel nut cannot be caused by improved visuospatial performance and memory. The three-location task does not require participants either to retain the to-beremembered items for later recall (memory) or to copy a complex figure (visuospatial performance). Rather, we have adopted the notion of attentional distribution to account for the object masking in the three-location task. Because the target in the three-location condition could appear in one of three locations randomly, spatial uncertainty was created. Therefore, the present study, as well as other, similar studies (e.g., Atchley et al., 2002; Enns, 2004; Enns \& Di Lollo, 1997), considers that the spatial attentional distribution over multiple target locations is a critical factor in such a task. In addition, many studies have shown that attentional distribution is associated with many other processes. For example, stimuli with a greater attentional distribution could have a faster processing rate (Carrasco \& McElree, 2001), increased spatial resolution (Carrasco, Williams, \& Yeshurun, 2002), and increased contrast appearance (Liu, Abrams, \& Carrasco, 2009). Hence, we suggest that in the conditions in which the object-masking effect was absent, attention was distributed more to the foveal location, leading to faster processing and better representation quality (e.g., increased spatial resolution and contrast appearance) of a target in that location.

One may be concerned that in the three-location condition, similar to that in Enns and Di Lollo (1997, Exp. 1), the different sensory effects caused by the different target locations (foveal or parafoveal) might confound the attentional effects (attended vs. unattended). This concern may be minor, for the following reason. If there were no attentional effects (attended vs. unattended), but only the sensory effects (foveal vs. parafoveal), object masking should be similar in both foveal and parafoveal target conditions, because object masking has been shown to be less sensitive to sensory effects (Enns, 2004; Enns \& Di Lollo, 1997). However, this was not the case in the present study; that is, object masking was more effective for the parafoveal target, but less effective for the foveal target (i. e., Cohen's $d$ was medium to large at the parafoveal target and small to medium at the foveal target). Thus, since even the object mask might have produced sensory masking to some extent, it is clear that additional, presumably attentional, masking occurred (Atchley et al., 2002).

Two general classes of models could account for masking (e.g., contour and object masking) in the present study. Although these two classes of models suggest that different mechanisms underlie masking, they do not prevent that in some cases, contour masking would be sensitive to sensory processing and object masking to attention. The first class of models argues that masking is related to disruption of reentrant (or recurrent) processing (e.g., Enns \& Di Lollo, 1997; Fahrenfort, Scholte, \& Lamme, 2007; Lamme \& Roelfsema, 2000; Lamme, Zipser, \& Spekreijse, 2002). In these models, a stimulus (e.g., a target) evokes transient feedforward processing and then later, reentrant processing back to the low levels. Only when the later, reentrant processing occurs is the stimulus sufficiently processed to allow for correct recognition. The masking effect might be accounted for by a mismatch in the feedforward processing of a mask and the reentrant processing of a target. Another 
class of models argues that backward masking is caused by feedforward lateral inhibition of a second stimulus (e.g., a mask) (e.g., Macknik \& Livingstone, 1998; Macknik \& Martinez-Conde, 2004, 2007). In these models, the transient responses to a mask's spatial edge and temporal edge could inhibit transient responses to a target's spatiotemporal edges, thus resulting in the invisibility of the target. Although the former class of models suggests reentrant processing to explain the masking effects, it also suggests that some masking paradigms (e.g., contour masking) cannot be explained by this reentrant processing (Fahrenfort et al., 2007). Instead, it is possible that lowlevel lateral inhibitory mechanisms (e.g., Macknik \& Livingstone, 1998; Macknik \& Martinez-Conde, 2004, 2007) might play a role when processing these types of stimuli. Also, although the latter class of models suggests feedforward lateral inhibition for the mask, it does not reject the importance of attention in object masking (e.g., Macknik \& Martinez-Conde, 2007). These models suggest that attention might be a distinct mechanism that modulates the neural circuits underlying masking (Macknik \& Martinez-Conde, 2007).

To conclude, betel nut chewing may immediately facilitate nonchewers' and habitual chewers' attention, but it does not affect their sensory processing. Furthermore, betel nut chewing strongly focuses nonchewers' attention, but it focuses habitual chewers' attention only moderately. Therefore, after chewing betel nuts, nonchewers and habitual chewers can pay more attention to foveal information (e.g., the car ahead of a bus driver). This facilitated foveal attention could possibly reduce the likelihood of car accidents. For example, if a bus driver's awareness is heightened, and he is consequently more attuned to noticing a car in front of the bus or to seeing a stop sign he otherwise might have missed, he might have more time to react appropriately to avoid an accident. However, because betel nut chewing affects the attention of nonchewers more than that of habitual chewers, this benefit of facilitated attention may be decreased when chewers become used to chewing betel nuts.

Author note This study was supported by Grant NSC-97-2410-H040-010 from the National Science Council. We thank Ying-Jui Ho, Hsin-Lun Huang, Shuo-Heng Li, Ya-Ling Shih, Wei-Chung Wang, Wan-Ting Hsieh, Hui-Tzu Kuo, Kai-Hui Hsu, Je-Fu Deng, and YuChi Chen for their assistance with the present study.

\section{References}

Asthana, S., Raffaele, K. C., Greig, N. H., Berardi, A., Morris, P. P., Schapiro, M. B., ... Soncrant, T. T. (1995). Neuroendocrine responses to intravenous infusion of arecoline in patients with Alzheimer's disease. Psychoneuroendocrinology, 20, 623-636.
Atchley, P., Grobe, J., \& Fields, L. M. (2002). The effect of smoking on sensory and attentional masking. Perception \& Psychophysics, 64, 328-336.

Atchley, P., \& Hoffman, L. (2004). Aging and visual masking: Sensory and attentional factors. Psychology and Aging, 19, 5767. doi:10.1037/0882-7974.19.1.57

Breitmeyer, B., \& Öğmen, H. (2006). Visual masking. New York: Oxford University Press.

Carrasco, M., \& McElree, B. (2001). Covert attention accelerates the rate of visual information processing. Proceedings of the National Academy of Sciences, 98, 5363-5367.

Carrasco, M., Williams, P. E., \& Yeshurun, Y. (2002). Covert attention increases spatial resolution with or without masks: Support for signal enhancement. Journal of Vision, 2, 4(6), 467-479. doi: $10.1167 / 2.6 .4$

Chen, C. R., Huang, Y. H., Song, H. C., \& Chang, S. R. (2003). Health survey for the long-distance bus drivers (IOSH92-M322). Taipei: Institute of Occupational Safety and Health.

Chiou, S. S., \& Kuo, C. D. (2008). Effect of chewing a single betelquid on autonomic nervous modulation in healthy young adults. Journal of Psychopharmacology, 22, 910-917.

Chu, N. S. (1994). Effects of betel chewing on electroencephalographic activity: Spectral analysis and topographic mapping. Journal of Formosa Medical Association, 93, 167-169.

Chu, N. S. (1995). Sympathetic response to betel chewing. Journal of Psychoactive Drugs, 27, 183-186.

Chu, N. S. (2001). Effects of betel chewing on the central and autonomic nervous system. Journal of Biomedical Science, 8 , 229-236.

Chu, N. S. (2002). Neurological aspects of areca and betel chewing. Addiction Biology, 7, 111-114.

Chu, N. S. (1993). Cardiovascular responses to betel chewing. Journal of Formosa Medical Association, 92, 835-837.

Clader, J. W., \& Wang, Y. (2005). Muscarinic receptor agonists and antagonists in the treatment of Alzheimer's disease. Current Pharmaceutical Design, 11, 3353-3361.

Cohen, J. (1988). Statistical power analysis for the behavioral sciences (2nd ed.). Hillsdale: Erlbaum.

Critchley, H. D. (2005). Neural mechanisms of autonomic, affective, and cognitive integration. Journal of Comparative Neurology, 493, 154-166.

Directorate-General of Budget, Accounting and Statistics. (1999). Survey on areca use in Taiwan. Taiwan: Taipei.

Enns, J. T. (2004). Object substitution and its relation to other forms of visual masking. Vision Research, 44, 1321-1331. doi:10.1016/ j.visres.2003.10.024

Enns, J. T., \& Di Lollo, V. (1997). Object substitution: A new form of masking in unattended visual locations. Psychological Science, 8 , 135-139. doi:10.1111/j.1467-9280.1997.tb00696.x

Enns, J. T., \& Di Lollo, V. (2000). What's new in visual masking? Trends in Cognitive Sciences, 4, 345-352.

Eysenck, M. W. (1982). Attention and arousal: Cognition and performance. Berlin: Springer.

Fahrenfort, J. J., Scholte, H. S., \& Lamme, V. A. F. (2007). Masking disrupts reentrant processing in human visual cortex. Journal of Cognitive Neuroscience, 19, 1488-1497.

Freo, U., Pizzolato, G., Dam, M., Ori, C., \& Battistin, L. (2002). A short review of cognitive and functional neuroimaging studies of cholinergic drugs: implications for therapeutic potentials. Journal of Neural Transmission, 109, 857-870.

Gupta, P. C., \& Warnakulasuriya, S. (2002). Global epidemiology of areca nut usage. Addiction Biology, 7, 77-83.

Haubrich, D. R., \& Watson, D. R. (1972). Effects of pilocarpine or arecoline administration on acetylcholine levels and serotonin turnover in rat brain. Journal of Pharmacology and Experimental Therapeutics, 181, 19-27. 
Hugdahl, K. (1996). Cognitive influences on human autonomic nervous system function. Current Opinion in Neurobiology, 6, $252-258$.

Hwang, L. S., Wang, C. K., \& Kao, L. S. (1993). Neuronal activity modulating components in betel quid. Kaohsiung, Taiwan: Symposium on Betel Quid Chewing and Its Health Effects.

Javed, F., Correa, F. O. B., Chotai, M., Tappuni, A. R., \& Almas, K. (2010). Systemic conditions associated with areca nut usage: A literature review. Scandinavian Journal of Public Health, 38, $838-844$

Lamme, V. A. F., \& Roelfsema, P. R. (2000). The distinct modes of vision offered by feedforward and recurrent processing. Trends in Neuroscience, 23, 571-579.

Lamme, V. A. F., Zipser, K., \& Spekreijse, H. (2002). Masking interrupts figure-ground signals in V1. Journal of Cognitive Neuroscience, 14, 1044-1053.

Laumann, K., Gärling, T., \& Stormark, K. M. (2003). Selective attention and heart rate responses to natural and urban environments. Journal of Environmental Psychology, 23, 125-134.

Lin, S. K., Chang, Y. J., Ryu, S. J., \& Chu, N. S. (2002). Cerebral hemodynamic responses to betel chewing: a Doppler study. Clinical Neuropharmacology, 25, 244-250.

Liu, T., Abrams, J., \& Carrasco, M. (2009). Voluntary attention enhances contrast appearance. Psychological Science, 20, 354-362.

Macknik, S. L., \& Livingstone, M. S. (1998). Neuronal correlates of visibility and invisibility in the primate visual system. Nature Neuroscience, 1, 144-149.

Macknik, S. L., \& Martinez-Conde, S. (2004). The spatial and temporal effects of lateral inhibitory networks and their relevance to the visibility of spatiotemporal edges. Neurocomputing, 58, $775-782$.

Macknik, S. L., \& Martinez-Conde, S. (2007). The role of feedback in visual masking and visual processing. Advances in Cognitive Psychology, 3, 125-152.

Molinengo, L., Fundaro, A. M., \& Cassone, M. C. (1988). Action of a chronic arecoline administration on mouse motility and on acetylcholine concentrations in the CNS. Journal of Pharmacy and Pharmacology, 40, 821-822.

Raffaele, K. C., Berardi, A., Haxby, J. V., Morris, P. P., Schapiro, M. B., \& Soncrant, T. T. (1996). Differential response to the cholinergic agonist arecoline among different cognitive modalities in Alzheimer's disease. Neuropsychopharmacology, 15, 163170.

Raffaele, K. C., Berardi, A., Morris, P. P., Asthana, S., Haxby, J. V., Schapiro, M. B., ... Soncrant, T. T. (1991). Effects of acute infusion of the muscarinic cholinergic agonist arecoline on verbal memory and visuo-spatial fuction in dementia of the Alzheimer type. Progress in Neuro-Psychopharmacology and Biological Psychiatry, 15, 643-648.

Ramachandran, V. S., \& Cobb, S. (1995). Visual attention modulates metacontrast masking. Nature, 373, 66-68.

Rinaldi, F., \& Himwich, H. E. (1955). Alerting responses and actions of atropine and cholinergic drugs. Archives of Neurology and Psychiatry, 73, 387-395.

Rosnow, R. L., \& Rosenthal, R. (1996). Computing contrasts, effect sizes, and counter nulls on other people's published data: General procedures for research consumers. Psychological Methods, 1, 331-340.

Stewart, A. L., \& Purcell, D. G. (1974). Visual backward masking by a flash of light: A study of U-shaped detection functions. Journal of Experimental Psychology, 103, 553-566.

Taylor, P. (1980). Cholinergic agonists. In A. G. Gilman, L. S. Goodman, \& A. Gilman (Eds.), The pharmacological basis of therapeutics (pp. 91-99). New York: Macmillan.

Von Euler, U. S., \& Domeij, B. (1945). Nicotine-like actions of arecoline. Acta Pharmacologica, 1, 263-269.

Winstock, A. (2002). Areca nut-Abuse liability, dependence and public health. Addiction Biology, 7, 133-138.

Winstock, A. R., Trivedy, C. R., Warnakulasuriya, K. A. A. S., \& Peters, T. J. (2000). A dependency syndrome related to areca nut use: Some medical and psychological aspects among areca nut users in the UK. Addiction Biology, 5, 173-179. 